\title{
GT2021-58907
}

\section{FLOW AND HEAT TRANSFER MECHANISMS IN A ROTATING COMPRESSOR CAVITY UNDER CENTRIFUGAL BUOYANCY-DRIVEN CONVECTION}

\author{
Feng Gao* \\ Fluid \& Acoustic Engineering Laboratory \\ Aero-Engine Research Institute \\ Beihang University \\ Beijing, 102206, P.R. China \\ Email: feng.gao@buaa.edu.cn
}

\author{
John W. Chew \\ Faculty of Engineering and Physical Sciences \\ University of Surrey \\ Guildford, GU2 7XH, United Kingdom \\ Email: j.chew@surrey.ac.uk
}

\begin{abstract}
This paper presents a systematic study of flow and heat transfer mechanisms in a compressor disc cavity with an axial throughflow under centrifugal buoyancy-driven convection, comparing with previously published experimental data. Wallmodelled large-eddy simulations are conducted for six operating conditions, covering a range of rotational Reynolds number $\left(3.2 \times 10^{5}-2.2 \times 10^{6}\right)$, buoyancy parameter $(0.11-0.26)$ and Rossby number (0.4-0.8). Numerical accuracy and computational efficiency of the simulations are considered. Wall heat transfer predictions are compared with measured data with a good level of agreement. A constant rothalpy core occurs at high Eckert number, appearing to reduce the driving buoyancy force. The flow in the cavity is turbulent with unsteady laminar Ekman layers observed on both discs except in the bore flow affected region on the downstream disc cob. The shroud heat transfer Nusselt number-Rayleigh number scaling agrees with that of natural convection under gravity for high Rayleigh numbers. Disc heat transfer is dominated by conduction across unsteady Ekman layers, except on the downstream disc cob. The disc bore heat transfer is close to a pipe flow forced convection correlation. The unsteady flow structure is investigated showing strong unsteadiness in the cavity that extends into the axial throughflow.
\end{abstract}

\section{NOMENCLATURE}

\section{Roman Symbols}

$a \quad$ Disc bore radius [m].

$b \quad$ Shroud radius [m].

$d \quad$ Axial gap between discs [m].

$d_{h} \quad$ Axial throughflow hydraulic diameter $=2\left(a-r_{s}\right)$.
Ec

$G r$

$R e_{a}$

Ro

$T$

$T_{r}$

$T^{*}$
Axial length of a disc bore [m].

Axial throughflow mass flow rate $\left[\mathrm{kg} \cdot \mathrm{s}^{-1}\right]$.

Amount of the axial throughflow exchanging with the cavity flow $\left[\mathrm{kg} \cdot \mathrm{s}^{-1}\right]$.

Radius [m].

Non-dimensional radius $=r / b$.

Shaft radius [m].

Velocity in the reference frame of the discs $\left[\mathrm{m} \cdot \mathrm{s}^{-1}\right]$.

Non-dimensional axial velocity $=v_{z} /(\Omega r)$.

Non-dimensional radial velocity $=v_{r} /(\Omega r)$.

Non-dimensional circumferential velocity $=v_{\theta} /(\Omega r)$.

Specific heat capacity at constant pressure $=1004.15$ $\left[\mathrm{J} \cdot \mathrm{kg}^{-1} \cdot \mathrm{K}^{-1}\right]$.

Eckert number $=(\Omega b)^{2} /\left(C_{p} \Delta T\right)$.

Grashof number $=R e_{\phi}^{2} \beta \Delta T$.

Rothalpy $=C_{p} T+0.5 \vec{v}^{2}-0.5(\Omega r)^{2}$.

Disc bore Nusselt number $=\frac{\dot{Q}_{a} d_{h}}{\kappa_{a}\left(T_{a}-\left\langle T_{\text {bulk }}\right\rangle\right)}$.

Shroud Nusselt number $=\frac{\dot{Q}_{b}(d / 2)}{\kappa_{\text {core }}\left(T_{r, b}-\left\langle T_{r, \text { core }}\right\rangle\right)}$.

Disc Nusselt number $=\frac{\dot{Q}_{d} r}{\kappa_{d}\left(T_{r, d}-T_{a}\right)}$.

Staic pressure $[\mathrm{Pa}]$.

Prandtl number $=0.7$.

Heat flux $\left[\mathrm{W} \cdot \mathrm{m}^{-2}\right]$.

Shroud Rayleigh number $=\frac{\rho_{\mathrm{core}}^{2} \Omega^{2} b\left(T_{r, b}-\left\langle T_{r, \text { core }}\right\rangle\right)(d / 2)^{3}}{\mu_{\text {core }}^{2}\left\langle T_{r, \text { core }}\right\rangle} \operatorname{Pr}$.

Rotational Reynolds number $=\frac{\rho \Omega b^{2}}{\mu}$.

Pipe flow Reynolds number $=\frac{\rho_{\text {bulk }} d_{h} \sqrt{W^{2}+(\Omega a)^{2}}}{\mu_{\text {bulk }}}$

Rossby number $=W /(\Omega a)$.

Static temperature [K].

Rotary stagnation temperature $=I / C_{p}$.

Non-dimensional temperature $=\left(T-T_{f}\right) / \Delta T$. 
$T_{r}^{*} \quad$ Non-dimensional rotary stagnation temperature $=\left(T_{r}-\right.$ $\left.T_{f}\right) / \Delta T_{r}$.

$W \quad$ Axial throughflow bulk velocity $\left[\mathrm{m} \cdot \mathrm{s}^{-1}\right]$.

$\mathscr{C}_{p}^{*} \quad$ Pressure coefficient $=\frac{P-\langle P\rangle}{0.5 \rho(\Omega b)^{2}}$.

\section{Greek Symbols}

$\beta \quad$ Thermal expansion coefficient $=1 / T_{f}$.

$\beta \Delta T$ Buoyancy parameter.

$\beta \Delta T_{r} \quad$ Actual driving buoyancy parameter.

$\delta \quad$ Laminar Ekman thickness $=\pi \sqrt{\mu /(\rho \Omega)}$.

$\Delta_{w} \quad$ Wall distance [m].

$\Delta_{w}^{*} \quad$ Non-dimensional wall distance $=\Delta_{w} / \delta$.

$\Delta T \quad$ Shroud-to-inlet temperature difference $=T_{b}-T_{f}$.

$\Delta T_{r} \quad$ Shroud-to-inlet rotary stagnation temperature difference $=T_{r, b}-T_{f}$.

$\kappa \quad$ Thermal conductivity $=\mu C_{p} / P r$.

$\mu \quad$ Dynamic viscosity $\left[\mathrm{kg} \cdot \mathrm{m}^{-1} \cdot \mathrm{s}^{-1}\right]$.

$\Omega \quad$ Disc angular speed [rad $\cdot \mathrm{s}^{-1}$ ] or $[\mathrm{rpm}]$

$\rho \quad$ Density $\left[\mathrm{kg} \cdot \mathrm{m}^{-3}\right]$.

\section{Subscripts}

a Disc bore.

$b$ Shroud.

core Core position.

$d \quad$ Disc.

$f \quad$ Inlet.

mid Mid-axial position.

rms Root mean square.

s Shaft.

$z, r, \theta$ Axial, radial and circumferential directions.

$\infty \quad$ Far-field.

\section{Other}

$\langle\cdot\rangle \quad$ Time- and circumferentially-averaged variable.

$\langle\cdot\rangle_{\text {rot }}$ Time average in the reference frame of the unsteady flow structure.

\section{Acronyms}

CFD Computational fluid dynamics.

LES Large-eddy simulation.

RANS Reynolds-averaged Navier-Stokes.

WMLES Wall-modelled LES.

\section{INTRODUCTION}

The need to reduce travel costs and environmental pollution drives development of turbofan engines to higher by-pass ratios. To achieve this smaller engine cores are required. However, unless blade tip clearances are also reduced the blade tip clearance-to-radius ratio becomes larger and this results in proportionally more severe tip clearance losses. As tip clearances are strongly influenced by disc thermal growth, understanding of the flow and heat transfer to the discs is required. Figure 1 illustrates rotating disc cavities typically seen in high pressure compressors, highlighting the cavity between the last two compressor stages. The disc cavity is bounded axially by adjacent disc diaphragms and radially by the shroud at the outer periphery. The cavity is open to the bore flow space that is formed between the disc bores and the driving shaft. Gas in the main annulus gains internal energy through the compression process and will heat up the shroud and the discs. The cavity is expected to be cooled by the axial throughflow that is directed from the low or intermediate pressure compressor main annulus. Though this kind of configuration is used in most modern gas turbines, in some applications a small amount of radial inflow bled through the shroud is shown to reduce the thermal time constants during an engine transient [1], while in other cases the cavity is sealed [2].

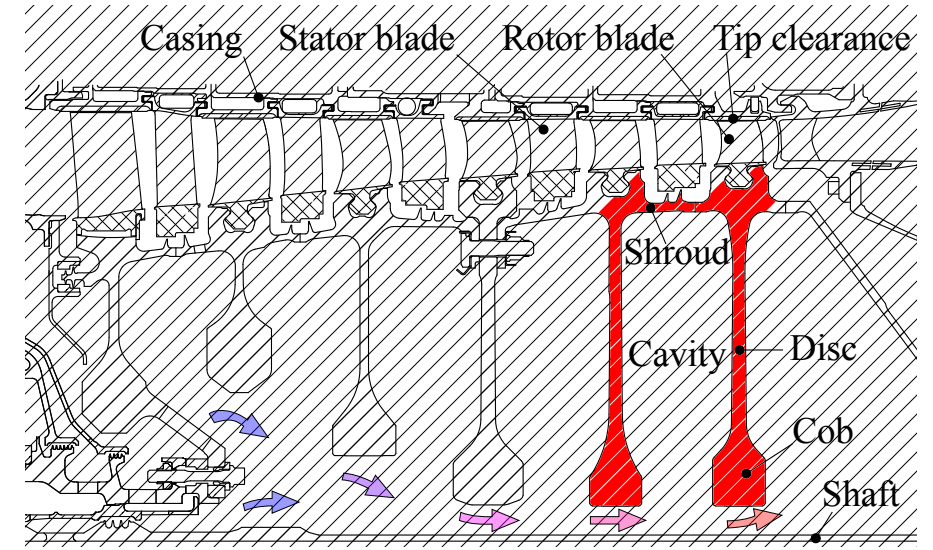

FIGURE 1. ROTATING CAVITIES IN A HIGH PRESSURE COMPRESSOR [3].

Known compressor disc cavity flow and heat transfer mechanisms were discussed in the review by Owen and Long [4]. Since then, a number of relevant studies have emerged [3,5-18]. A complete review is not attempted here, but a short account is given to illustrate previous work towards understanding and predicting the general flow structure, the shroud heat transfer, the main disc boundary layers and heat transfer, and heat transfer on the cobs and disc bore.

The flow in rotating cavities is dominated by centrifugal buoyancy-driven convection, and is 3-D, unsteady and unstable.

Owen and co-workers $[4,10,15]$ assumed laminar fluid core in the cavity and developed simple models based on this hypothesis. However, Long et al.'s [19] unsteady velocity measurements showed evidence of turbulent fluctuations in the cavity core. The large-eddy simulations of Sun et al. [20] and Pitz et al. [17] show large scale unsteady flow structures dominate the cavity core and the velocity spectra follow the $-5 / 3$ slope for turbulent flow. In terms of core temperature uniform static temperature profiles were obtained by Pitz et al. [6] and Gao et al. [9], while Saini and Sandberg's [13] study shows the core temperature increasing slightly with radius. This may be due to Saini and Sandberg's simulation having a higher Mach number [6,9]. The increase of static temperature with radius is attributed to the compressibility effect and considered to cause possible reduction in the shroud heat transfer at higher rotational Rayleigh numbers.

The shroud heat transfer in rotating cavities shows a strong 
analogy with that of horizontal plates under gravity. When the Rayleigh number is greater than a critical value, the shroud heat transfer is by convection [5]. Considering this analogy Long and Childs [21] reported using cavity core temperature, instead of inlet flow temperature, as the most appropriate to correlate the measured mean shroud Nusselt number. Using the cavity core temperature as reference Gao and Chew [3] correlated their numerically predicted mean shroud Nusselt number with the Rayleigh number, and showed good agreement with a correlation of natural convection between horizontal plates under gravity for high Rayleigh numbers. Further to this Puttock-Brown et al. [7] and Saini and Sandberg [11] reported Rayleigh-Bénard streaks near the shroud.

Farthing et al.'s [22] experimental flow visualisation showed the formation and breakdown of toroidal vortices in the presence of an axial throughflow. They also reported that the cold fluid entering and the hot fluid leaving the cavity are confined within Ekman layers. From a recent published experimental study Jackson et al. [10] conclude that the hot fluid leaves the cavity within the Ekman layer and mixing with the axial throughflow occurs at the low-radius region of the cavity. Based on this steady Ekman layer flow assumption Owen et al. [15] developed a series of simple models to correlate existing heat transfer data. Saini and Sandberg's [11] unsteady simulation confirms the existence of the toroidal vortex at high Rossby numbers. However, Saini and Sandberg's work shows outward mean radial flow through the disc Ekman layer, in contrast to Owen and co-workers' Ekman layer radial inflow assumption. Pitz et al. [6] and Gao et al.'s [9] direct and large-eddy simulations of a sealed cavity show averaged Ekman layer radial in- and outflow at different radii depending on the sign of the circumferential core slip, but the time dependent Ekman layer is laminar and highly unsteady.

Due to the presence of the Coriolis force in the rotating frame of reference the Ekman layer can retain a laminar state at very high rotational Rayleigh numbers. The studies of Pitz et al. [6], Gao et al. [9] and Saini and Sandberg all show unsteady laminar disc Ekman layers. The disc heat transfer mechanisms have been considered by Pitz et al. [17] and Saini and Sandberg [11], but no definitive conclusion was obtained.

The heat transfer between the disc bore and the axial throughflow is usually unreported. This is considered in the simple model of Tang et al. [14], but no validation against experimental data or CFD (computational fluid dynamics) prediction was presented.

It should also be noted that published unsteady simulations for real engine representative geometries [11,20,23] only considered relatively high Rossby number $(\geq 0.44)$.

The above literature review indicates a need for clarification of flow and heat transfer mechanisms at engine operating conditions. This paper aims to prove a systematic description of the flow and heat transfer mechanisms in an engine representative compressor cavity at a steady operating condition. The main text is organised as follows. The rig geometry and operating conditions considered are described in Section 2. The numerical model and set up are presented in Section 3, followed by a comprehensive evaluation against measured data. Section 4 gives a systematic description of the flow and heat transfer mechanisms in the rotating compressor cavity. Finally, the main findings are summarised in Section 5.

\section{DESCRIPTION OF THE CONFIGURATIONS STUD- IED}

The present work considers the rotating compressor cavity rig recently established at the University of Bath [10,18], and shown in Fig. 2. The model compressor drum is rotating, whilst the shaft is stationary. Three disc cavities are formed between four disc diaphragms. For the experiments considered cavities 1 and 3 are sealed at the disc bore and thermally insulated. This is to focus on the physics of a single rotating cavity, i.e. cavity 2 . The shroud is heated. The stationary shaft is assumed here to be adiabatic. An axial throughflow is introduced between the disc bore and the shaft. The radii of the shroud, disc bore and shaft are, respectively, $0.24 \mathrm{~m}, 0.07 \mathrm{~m}$ and $0.052 \mathrm{~m}$. The axial distance between discs 2 and 3 is $0.04 \mathrm{~m}$. Further information regarding the rig and the instrumentation can be found in Ref. [18].

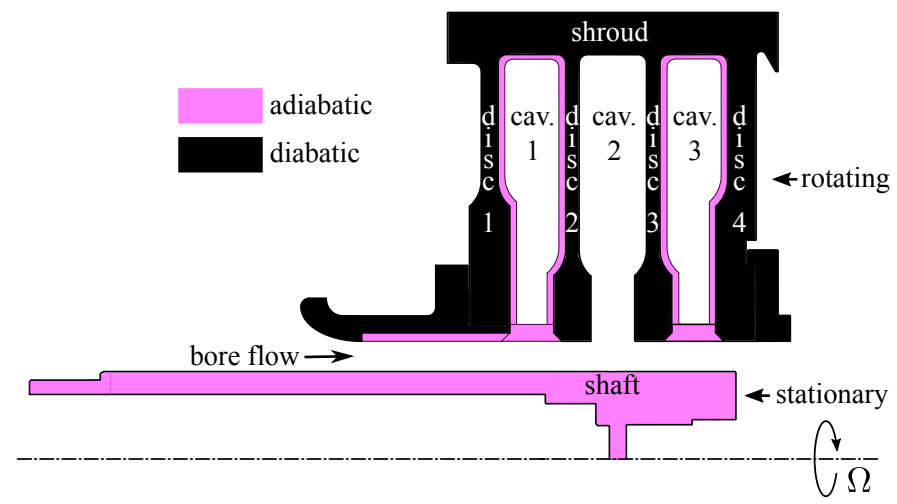

FIGURE 2. ROTATING COMPRESSOR CAVITY RIG RECENTLY ESTABLISHED AT THE UNIVERSITY OF BATH [18].

Six operating conditions are considered in the present study, covering a range of rotational Reynolds number $\left(R e_{\phi}=3.2 \times\right.$ $\left.10^{5}-2.2 \times 10^{6}\right)$, buoyancy parameter $(\beta \Delta T=0.11-0.26)$ and Rossby number $(R o=0.4-0.8)$. Some parameters for the operating conditions are given in Tab. 1 . The definitions of nondimensional parameters are given in Nomenclature at the beginning of this paper.

\section{NUMERICAL SET-UP AND VALIDATION}

\subsection{Solver}

The Rolls-Royce proprietary CFD code, Hydra, is used in this study. Hydra solves the compressible Navier-Stokes equations on unstructured meshes using the control volume method. A modified Roe scheme is considered for spatial discretisation. Second order spatial accuracy is guaranteed with a linear reconstruction of primitive variables at the grid cell interface to calculate fluxes [24]. An explicit three-step Runge-Kutta method is 
TABLE 1. OPERATING CONDITIONS.

\begin{tabular}{lllllll}
\hline Code & $\Omega[\mathrm{rpm}]$ & $\beta \Delta T$ & $R o$ & $10^{-5} R_{\phi}$ & $10^{-10} \mathrm{Gr}$ & $E c$ \\
\hline 800B11 & 790 & 0.11 & 0.5 & 3.2 & 1.1 & 0.012 \\
800B20 & 790 & 0.2 & 0.5 & 3.2 & 2.1 & 0.007 \\
800B26 & 790 & 0.26 & 0.5 & 3.2 & 2.6 & 0.005 \\
2kR4 & 2000 & 0.25 & 0.4 & 7.9 & 16 & 0.034 \\
2kR8 & 2000 & 0.25 & 0.8 & 8 & 16 & 0.034 \\
6kR4 & 6000 & 0.24 & 0.4 & 22 & 120 & 0.312 \\
\hline
\end{tabular}

considered as temporal scheme. For further information of the Hydra solver readers are referred to Ref. [24].

Unsteady simulations of internal air system flows usually require an order of magnitude more computational resources than those of main annulus flows. To achieve affordable computational cost wall-modelled large-eddy simulation (WMLES) is used here. The Smagorinsky subgrid-scale model with von Driest damping is employed to simulate subgrid flow motions. Simple functions representing the law of the wall are implemented at near-wall mesh points for the momentum and energy equations [25].

The performance in use of WMLES for internal air systems has been proved successful in recent studies [26,27]. It should be noted that there is a compromise between accuracy and computing requirements. In the present study the $6000 \mathrm{rpm}$ condition has consumed $8 \times 10^{5} \mathrm{CPU}$ hours on 1120 Xeon Gold $6148 \mathrm{~F}$ CPU cores. Fully resolved LES would require an order of magnitude higher computing time. Results for a sealed rotating cavity in Ref. [27] show consistency of WMLES with DNS [16] and fully resolved LES [9] at lower Rayleigh numbers, and extend calculations to high Rayleigh numbers.

\subsection{Model}

A full $360^{\circ}$ model is considered in this study. An example of the WMLES mesh on the meridional plane for the $6000 \mathrm{rpm}$ condition is given in Fig. 3. The inlet is set $3.8 d_{h}$ upstream of the upstream disc cob, while the outlet is placed $4.2 d_{h}$ downstream of the downstream disc cob. The near wall grid spacing is defined following the approach presented in Ref. [27], i.e. the minimum value of the Ekman depth $(\sqrt{\mu /(\rho \Omega)})$ and an a priori estimated spacing for $y^{+}=50$. The total grid point numbers for the 790, 2000 and $6000 \mathrm{rpm}$ conditions are, respectively, $\sim 2.3 \times 10^{6}$, $\sim 9 \times 10^{6}$ and $\sim 2.8 \times 10^{7}$, with 400,675 and 1000 nodes in the circumferential direction. The a posteriori $y^{+}$is, taking the $6000 \mathrm{rpm}$ condition as example, $\sim 5$ at the shroud and increases gradually, along the disc surface, to $\sim 30$ at the disc cob region. Finer grid resolutions have been used for the other lower speed conditions. The grid quality in the core is restricted by the mesh requirement on the shroud and disc surface, i.e. $z^{+}$or $r^{+}$, which cannot be significantly relaxed. Therefore, in the core the grid quality is close to that in a wall-resolved LES.

The flow through the bellmouth was simulated using the Spalart-Allmaras RANS model to obtain the inlet swirl ratio for

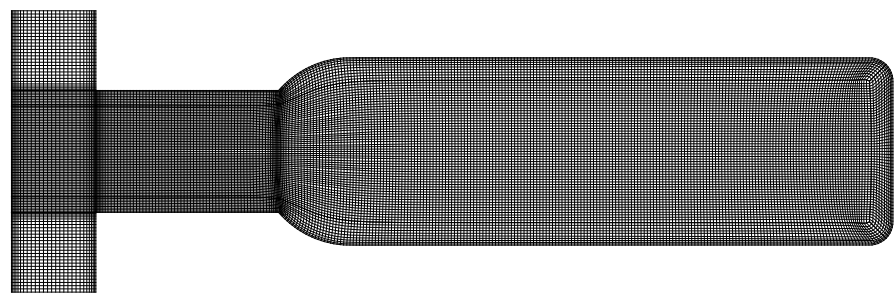

FIGURE 3. EXAMPLE OF THE BATH RIG SIMULATION MESH FOR 6000 RPM.

the main calculation. This is subsequently defined as the inlet boundary condition along with total pressure, and total temperature. The inlet temperature is the average of those measured on the rake underneath the upstream disc cob (shown in Fig. 6), and the variation in temperature at this position was no more than $\sim 1 \%$ of the inlet-to-shroud temperature difference. For the main disc cavity simulation the outlet employs a non-reflective condition at ambient pressure. The inlet total pressure was adjusted to approximately match the experimental mass flow rate. No-slip conditions are set on the solid walls with the corresponding angular speeds. Measured temperatures [10,28] are specified on the shroud, discs and the disc bores. The shaft and the other surfaces at the disc bore radius are assumed to be adiabatic.

All simulations presented in this paper are initialised with steady wall-modelled RANS solutions. A first 100 rotor revolutions are used to reach the steady state in the WMLES, and an additional 100 revolutions are simulated for collecting statistics.

\subsection{Validation}

Prior to detailed analysis of the flow mechanisms the accuracy of the simulations is considered in this section. An example of the shroud non-dimensional near-wall mesh spacing $y^{+}$and the contribution of the wall function to the shroud heat flux is given in Fig. 4 for the $6000 \mathrm{rpm}$ condition. The wall function contributes slightly $(\sim 4 \%)$ to the shroud heat flux. As shown below the discs are almost adiabatic at lower radii, and so the wall function has little effect on heat transfer on the disc faces and shroud. The balances of the mass flow rate, angular momentum and energy are checked for all test cases, and are given in Tab. 2. Very good mass balance is obtained, and the moment balance based on the rotor torque is satisfactory. The ratio of the energy imbalance to the total wall heat flux is satisfactory but gets larger at low $\beta \Delta T$ or high $R o$ conditions. This may be associated with the coarse mesh resolution in the WMLES.

Table 3 compares the WMLES solutions with the experimental mass flow rates and shroud heat transfer [28]. In WMLES the inlet total pressure is adjusted to approximately match the measured mass flow rate. Satisfactory agreement of the mean shroud heat flux between the experiment and the WMLES is achieved for the three high speed conditions, whilst larger differences of up to $24 \%$ are found for low speed conditions. An example of the disc heat transfer is given in Fig. 5 for the $=6000$ rpm condition, showing a good level of agreement between the WMLES solutions and the Bath estimates of heat flux (derived from disc temperature measurements [10]). Some difference of 
up to $\sim 20 \%$ between the WMLES and the experiment appears at $r^{*}=\sim 0.9$, but reduces as the shroud is approached. Some other differences are seen near the cobs as the geometrical changes are reflected in the simulation but not fully accounted for in the experimentally derived values. Note that, as reported in Ref. [10], there is significant uncertainty in the experimentally-derived values.
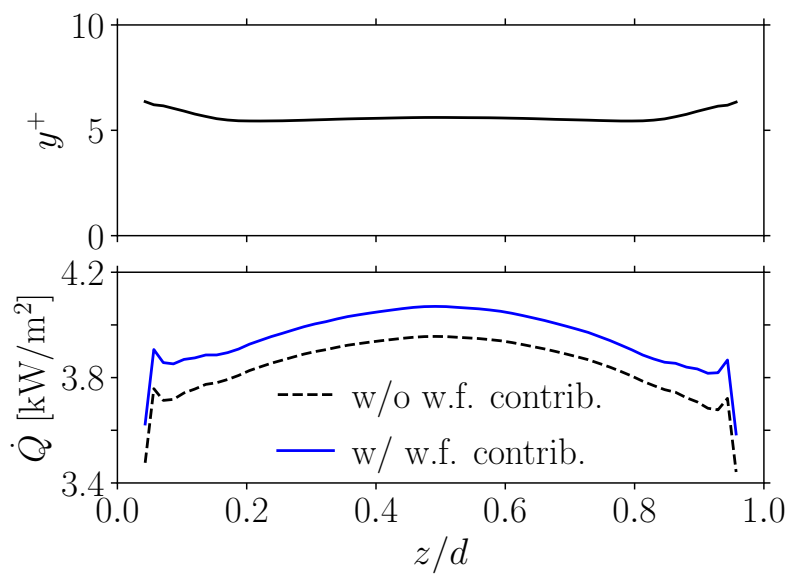

FIGURE 4. SHROUD $y^{+}$AND HEAT FLUX DISTRIBUTION FOR 6000 RPM.

TABLE 2. BALANCE CHECK.

\begin{tabular}{llll}
\hline Code & Mass flow rate & Angular momentum & Energy \\
\hline $800 \mathrm{~B} 11$ & $0.37 \%$ & $9.7 \%$ & $22.9 \%$ \\
$800 \mathrm{~B} 20$ & $0.44 \%$ & $7.3 \%$ & $12.5 \%$ \\
$800 \mathrm{~B} 26$ & $0.46 \%$ & $5.6 \%$ & $9.4 \%$ \\
$2 \mathrm{kR} 4$ & $0.40 \%$ & $2.5 \%$ & $10.3 \%$ \\
$2 \mathrm{kR} 8$ & $0.25 \%$ & $0.79 \%$ & $18.0 \%$ \\
$6 \mathrm{kR} 4$ & $0.04 \%$ & $6.2 \%$ & $12.4 \%$ \\
\hline
\end{tabular}

The mean static temperatures in the bore flow were measured at two axial locations between the cobs and the shaft, as given in the left subplot of Fig. 6. The comparison between the WMLES and the measurement [10], for $6000 \mathrm{rpm}$, is shown in the right subfigure of Fig. 6. The difference in temperature rise from upstream to downstream position may be associated with higher shroud heat flux in the experiment. However both WMLES and experimental results indicate heating of the air upstream of the cavity. As discussed further below this is associated with large scale flow unsteadiness that will be sensitive to experimental and numerical boundary conditions.

Unsteady flow structures are captured in both the WMLES and the experiment [29]. The present simulations predict unsteady flow features rotating at $84-89 \%$ of the disc angular speed, in close agreement with the experiment. However, the amplitudes of the pressure coefficient are more pronounced in the WMLES.
The validation presented above indicates the overall performance of the WMLES is satisfactory. An accuracy level of 20\% in heat transfer predictions would be considered good in many engineering applications. Some numerical inaccuracies occur in the present models as a result of compromises between computational requirements and accuracy. The numerical errors are of similar magnitude to differences between predictions and measurements, and to uncertainties associated with boundary conditions. The following sections focus on interpretation of the solutions to give understanding of the flow and heat transfer mechanisms and hence inform more elementary modelling approaches that are typically used in industry.

TABLE 3. COMPARISON BETWEEN THE WMLES AND EXPERIMENT [28].

\begin{tabular}{lllrr}
\hline \multirow{2}{*}{ Code } & \multicolumn{2}{c}{ Mass flow rate $\left[\mathrm{kg} \cdot \mathrm{s}^{-1}\right]$} & \multicolumn{2}{c}{ Shroud total heat } \\
& Exp. & WMLES & Diff. & WMLES-Exp. Diff. \\
\hline $800 \mathrm{~B} 11$ & 0.024 & 0.025 & $4.2 \%$ & $-20.9 \%$ \\
$800 \mathrm{~B} 20$ & 0.024 & 0.024 & $0 \%$ & $-22.4 \%$ \\
$800 \mathrm{~B} 26$ & 0.024 & 0.024 & $0 \%$ & $-24.0 \%$ \\
$2 \mathrm{kR} 4$ & 0.049 & 0.050 & $2.0 \%$ & $-14.1 \%$ \\
$2 \mathrm{kR} 8$ & 0.098 & 0.099 & $1.0 \%$ & $-11.9 \%$ \\
$6 \mathrm{kR} 4$ & 0.136 & 0.141 & $3.7 \%$ & $-13.1 \%$ \\
\hline
\end{tabular}
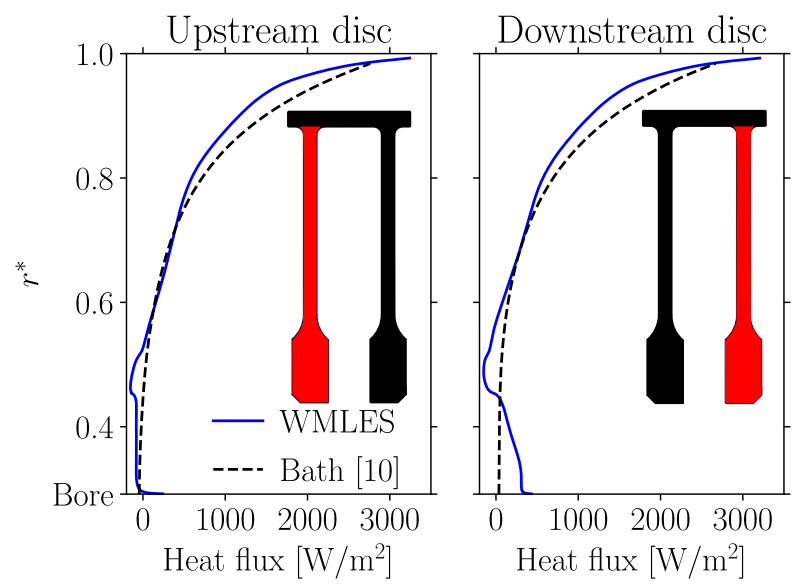

FIGURE 5. MEAN DISC HEAT TRANSFER FOR 6000 RPM. COURTESY OF UNIVERSITY OF BATH [10].

\section{FLOW AND HEAT TRANSFER MECHANISMS}

This section considers characteristics of the unsteady flow and offers a comprehensive view of the flow and heat transfer mechanisms in the cavity core, the shroud boundary layer, near the discs, and beneath the cobs.

\subsection{Shroud and core}

Normalised mean tangential velocity and the corresponding fluctuation profiles at the cavity mid-axial position are plotted in 


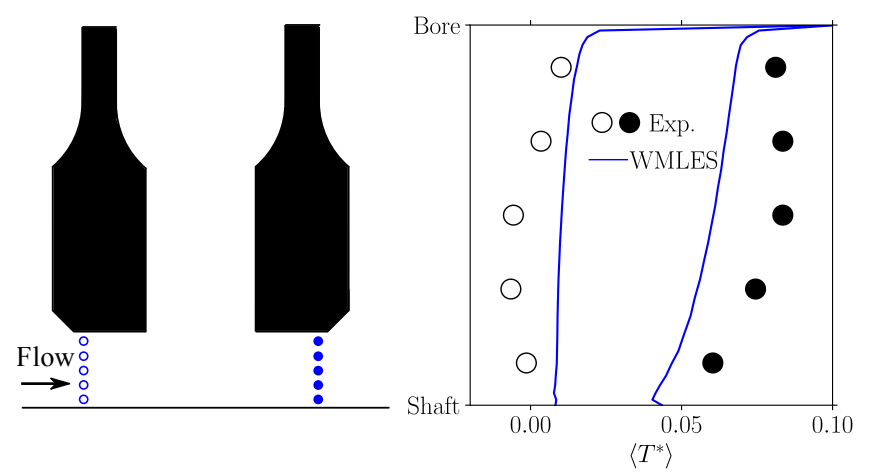

FIGURE 6. BORE FLOW TEMPERATURE FOR 6000 RPM. COURTERSY OF UNIVERSITY OF BATH [10].

Fig. 7. In this and other figures the shaft and disc bore positions are marked "S" and "B". The mean tangential velocities show a negative core slip and a clear Rossby number effect. Note, however, that positive core slip may occur in multiple cavity configurations, where the bore flow can take swirl from the upstream cavity $[19,30]$. For the tangential velocity fluctuation a local peak is observed near the shroud indicating the kinematic boundary layer, while stronger fluctuation appears at the radial location of the disc bore due to the strong interaction between the bore and cavity flows. A clear scaling is shown as the tangential velocity fluctuation is further normalised with $\sqrt{\beta \Delta T}$, consistent with the previous study in a sealed cavity [9]. $\Omega r \sqrt{\beta \Delta T}$ is also referred to as free-fall velocity in the geophysics research community.

Radial velocity profiles are shown in Fig. 8. The mean radial velocity changes sign near the disc bore radius, consistent with flow impingement on the downstream cob and formation of a toroidal vortex. A Rossby number effect is also observed. The radial velocity fluctuation (not shown here) scales with $\Omega r \sqrt{\beta \Delta T}$ for $r^{*}>0.5$.

In order to show the flow state in the cavity core the turbulent kinetic energy (TKE) spectra, defined as half of the sum of the power spectral density (PSD) in the three directions, were recorded at the positions shown in the inset of Fig. 9. The left subplot in the figure shows the lowest Grashof number condition, while the right subfigure presents the highest $G r$ condition considered in the present study. All spectra plotted show curves with clear $-5 / 3$ slope, indicating the flow is turbulent. The flow at the bore and cob region shows stronger unsteadiness than in the centre of the cavity. The increasing level of turbulence at high $G r$ is associated with trends in the disc heat transfer as further discussed in section 4.2.

For the $6000 \mathrm{rpm}$ condition the Eckert number, as shown in Tab. 1 , is 0.312 . This indicates that the kinetic energy associated with rotation is significant when compared to changes in internal energy. The dashed curves shown in Fig. 10 correspond to the mean static temperatures for medium and high rotational speed conditions. A uniform mean static temperature profile is observed in the core for the $2000 \mathrm{rpm}$ condition, whereas that for $6000 \mathrm{rpm}$ rises with radius due to rotational effects. In a rotating frame of reference rotary stagnation enthalpy or rothalpy [31] is given by
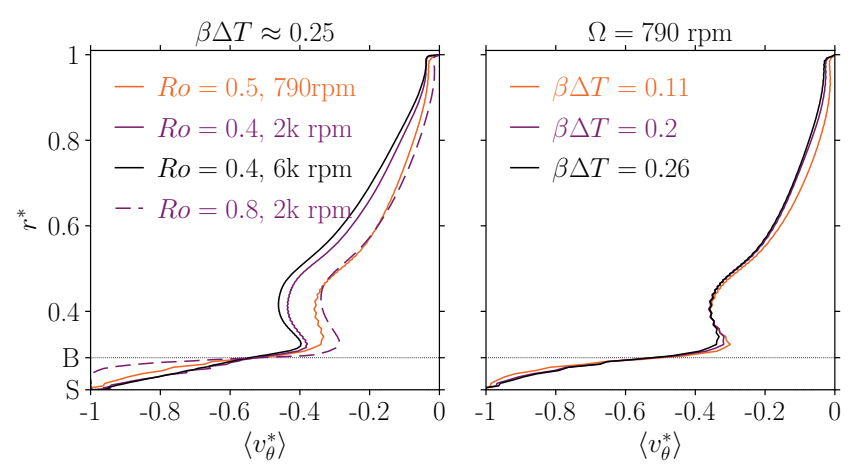

(a) Mean
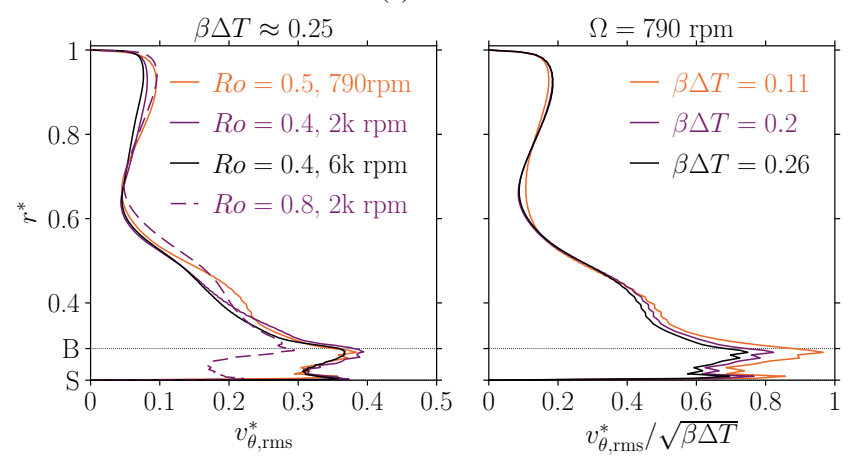

(b) Fluctuation

FIGURE 7. MEAN TANGENTIAL VELOCITY AND ITS FLUCTUATION PROFILES AT THE CAVITY MID-AXIAL POSITION. "S" AND "B" INDICATE RESPECTIVELY SHAFT AND DISC BORE POSITIONS.
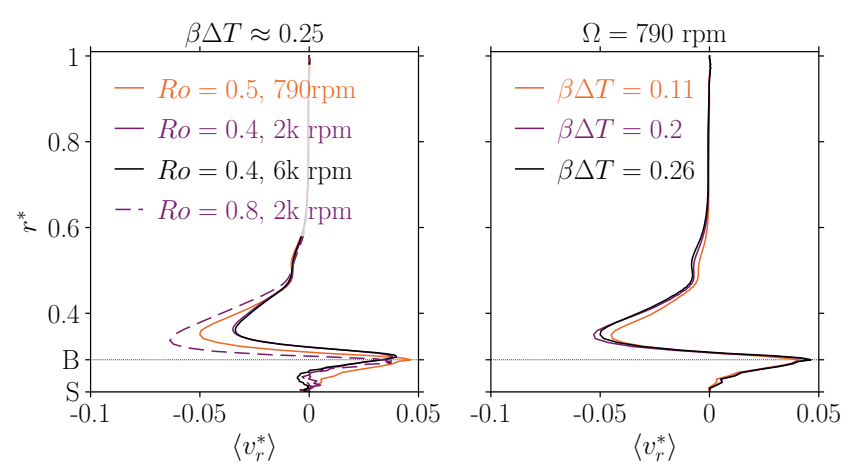

FIGURE 8. MEAN RADIAL VELOCITY PROFILE AT THE CAVITY MID-AXIAL POSITION.
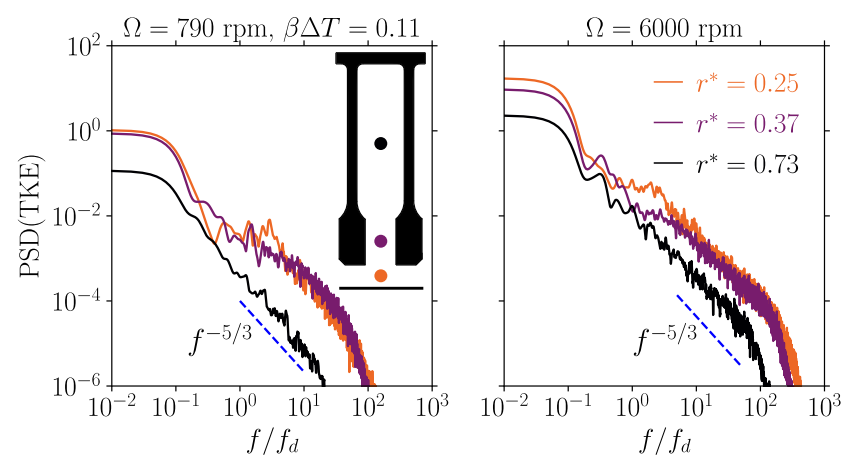

FIGURE 9. VELOCITY SPECTRA. SAMPLING LOCATIONS ARE SHOWN IN THE INSET. 


$$
I=C_{p} T+0.5 \vec{v}^{2}-0.5(\Omega r)^{2} .
$$

Rothalpy is comparable to total enthalpy in a stationary system. It is conserved along streamlines in steady, inviscid, adiabatic flow and conserved in steady flow systems with no net heat transfer or work done in the rotating frame. The rotary stagnation temperature, defined as

$$
T_{r}=I / C_{p},
$$

is also plotted in Fig. 10. Both conditions show uniform $\left\langle T_{r}^{*}\right\rangle$ profiles within the cavity. This confirms a uniform mean rothalpy core flow, consistent with the previous study for a sealed cavity [27]. Equations (1) and (2) show that the difference between $T$ and $T_{r}$ is determined by the relative fluid velocity and the tangential velocity of the disc at the same radius. Given that the core slip is small as shown in Fig. 7 the term dominating the difference is the disc dynamic head $0.5(\Omega r)^{2}$. Considering the temperature difference between the inflow and the shroud, $\Delta T$, the parameter affecting the difference between the profiles of $\left\langle T^{*}\right\rangle$ and $\left\langle T_{r}^{*}\right\rangle$ is the Eckert number $E c=(\Omega b)^{2} /\left(C_{p} \Delta T\right)$. Considering the rotary stagnation temperature the actual driving $\beta \Delta T_{r}$ for the $6000 \mathrm{rpm}$ condition is 0.2 , i.e. $16.7 \%$ smaller than using the static temperature.
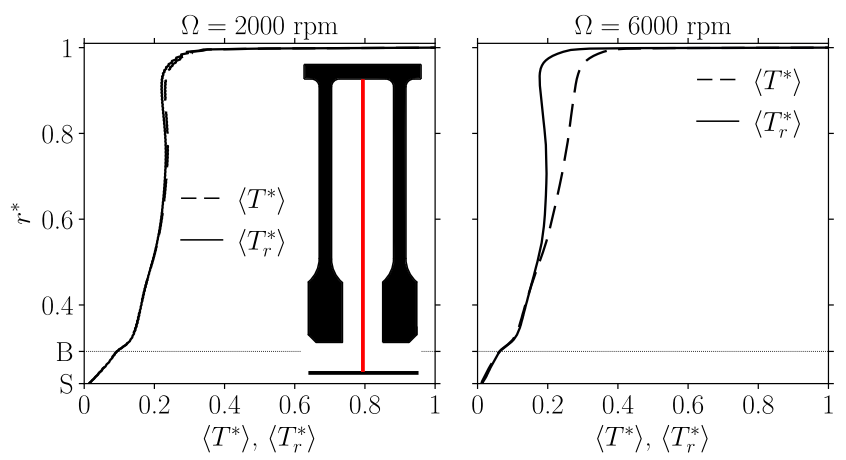

FIGURE 10. MEAN STATIC AND ROTARY STAGNATION TEMPERATURES AT THE CAVITY MID-AXIAL POSITION. $\Omega=2000$ RPM AND $6000 \mathrm{RPM}, R o=0.4$.

The mean rotary stagnation temperature profiles for all cases, averaged both in time and the circumferential direction, are plotted in Fig. 11. The uniform core $\left\langle T_{r}^{*}\right\rangle$ is shown for all conditions considered, as expected. For conditions with the same $\beta \Delta T$ increasing the disc angular speed results in lower $\left\langle T_{r}^{*}\right\rangle$. With the same $\beta \Delta T$ and rotational speed $\left\langle T_{r}^{*}\right\rangle$ rises with $R o$. A particularly sharp $\left\langle T_{r}^{*}\right\rangle$ change occurs at the disc bore radius for the high $R o$ condition. At fixed disc angular speed and $R o$ the two conditions with higher $\beta \Delta T$ show similar $\left\langle T_{r}^{*}\right\rangle$ profiles, which are slightly lower than for the low $\beta \Delta T$ condition. Referring again to Fig. 11, the rotary stagnation temperature fluctuation presents two local peaks with the outer one corresponding to the shroud thermal boundary layer and the inner one associated with the interaction between the cavity and bore flows. Similar fluctuation amplitude at the shroud thermal boundary layer is observed at all conditions studied.
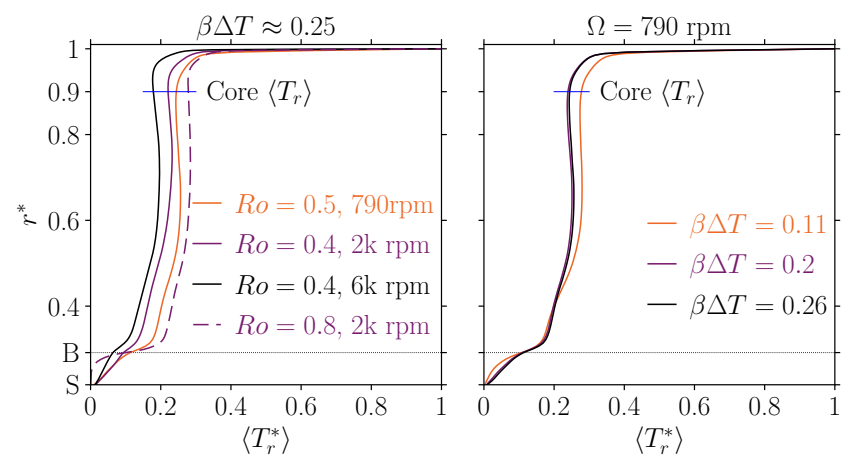

(a) Mean rotary stagnation temperature
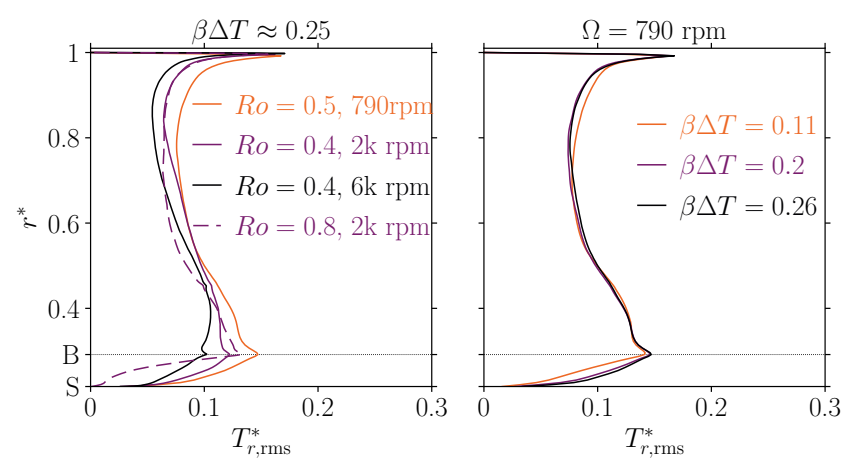

(b) Rotary stagnation temperature fluctuation

FIGURE 11. ROTARY STAGNATION TEMPERATURES AT THE MID-AXIAL POSITION FOR ALL CONDITIONS CONSIDERED.

The shroud $N u_{b} \sim R a^{n}$ scaling is considered in Fig. 12, with the data obtained in the present study and previous work for sealed and open Aachen rig geometries [3,27]. The definitions of the shroud $N u_{b}$ and $R a$ (based on the core-to-surface temperature difference) adapt those for natural convection above a heated horizontal plate under gravity:

$$
\begin{gathered}
R a=\frac{\rho_{\text {core }}^{2} \Omega^{2} b\left(T_{r, b}-\left\langle T_{r, \text { core }}\right\rangle\right)(d / 2)^{3}}{\mu_{\text {core }}^{2}\left\langle T_{r, \text { core }}\right\rangle} P r \\
N u_{b}=\frac{\dot{Q}_{b}(d / 2)}{\kappa_{\text {core }}\left(T_{r, b}-\left\langle T_{r, \text { core }}\right\rangle\right)} .
\end{gathered}
$$

where the core value is obtained at the mid-axial position in the cavity at $r^{*}=0.9$ as shown in Fig. 11, and is chosen to be outside the boundary layer in the constant rothalpy core. In Fig. 12 the high $R a$ data show very good consistency between different configurations and conditions.

Fitting all data (from the present study and previous work $[3,27])$ leads to the correlation $N u_{b}=0.057 R a^{0.366}$. This correlation is compared with those of natural convection between horizontal plates under gravity on the same plot and fair agreement is achieved with the high Rayleigh number correlation.

\subsection{Disc boundary layers}

The mean and instantaneous characteristics of the disc Ekman layers and the mean disc heat transfer are considered here. 


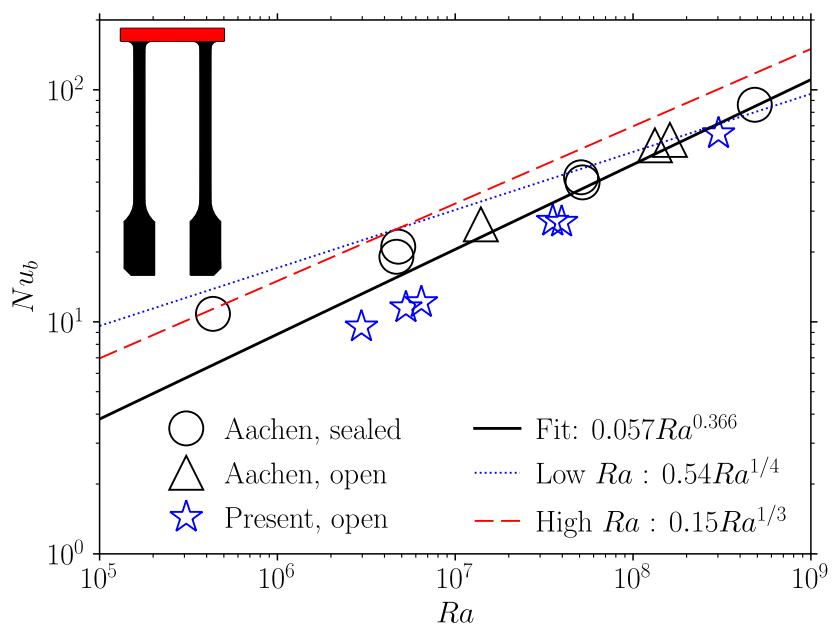

FIGURE 12. SCALING OF MEAN SHROUD NUSSELT NUMBER.

Figure 13 shows time and circumferentially averaged radial velocity, radial velocity fluctuation and rotary stagnation temperature profiles for both the up and downstream disc boundary layers. The non-dimensional wall distance to the disc $\Delta_{w}^{*}$ is defined as the ratio of the dimensional wall distance $\Delta_{w}$ to the thickness of the laminar Ekman layer $\delta=\pi \sqrt{\mu /(\rho \Omega)}$. The mean radial velocity and the corresponding fluctuations show laminar Ekman layers on both discs for $r^{*} \geq 0.6$. Weak, mean radial outflow in the Ekman layers is observed for all radial locations plotted, associated with the negative core slip as shown in Fig. 7. The fluctuations are large compared to the mean values, confirming the dominance of unsteady effects, and that radial transport of fluid in the Ekman layers is small compared to that in the cavity core. At higher radii, where disc heat flux is significant, the rotary stagnation temperature varies steeply across the laminar Ekman layer, as shown in the subplot (c). This supports the interpretation of the disc heat transfer across the laminar Ekman layer given later in this section.

The above discussion confirms the presence of unsteady laminar Ekman layers on the discs. Here the instantaneous disc boundary layer velocity profile is evaluated against the theoretical equation for an unsteady laminar Ekman layer, as given in Eqs. (5) and (6).

$$
\begin{aligned}
& v_{r}=v_{r, \infty}\left(1-e^{-\Delta_{w} \pi / \delta} \cos \frac{\Delta_{w} \pi}{\delta}\right)-v_{\theta, \infty} e^{-\Delta_{w} \pi / \delta} \sin \frac{\Delta_{w} \pi}{\delta} \\
& v_{\theta}=v_{\theta, \infty}\left(1-e^{-\Delta_{w} \pi / \delta} \cos \frac{\Delta_{w} \pi}{\delta}\right)+v_{r, \infty} e^{-\Delta_{w} \pi / \delta} \sin \frac{\Delta_{w} \pi}{\delta}
\end{aligned}
$$

Comparison of the instantaneous velocity profiles between the WMLES solution and the Ekman solution is shown in Fig. 14. The time instant presented corresponds to the snapshot in Fig. 19 (c) and the 3D flow field is discussed later. The circumferential positions in Fig. 14 are selected to show representative radial velocities, with positive, negative and near-zero components. The origin $\left(0^{\circ}\right)$ is at the 3 o'clock position in Fig. 19 (c) and the angle increases in the anti-clockwise direction. Despite the limited

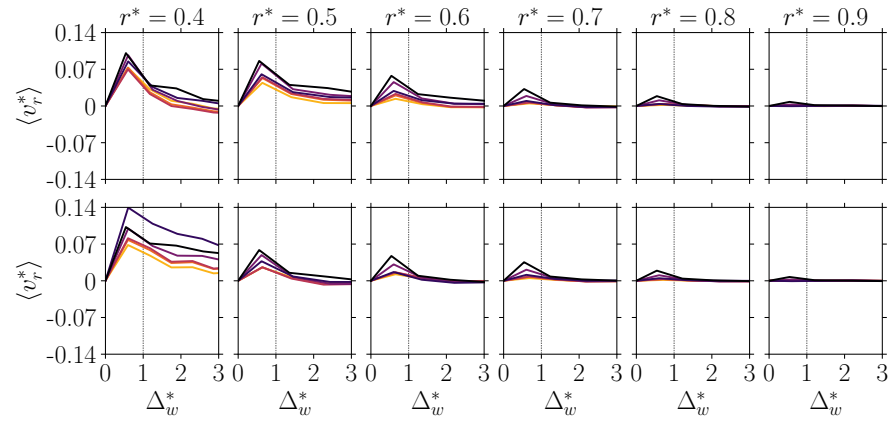

(a) Mean radial velocity

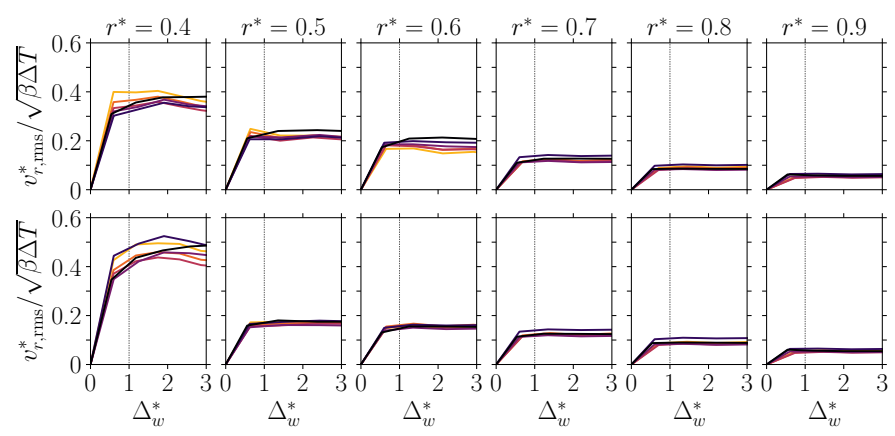

(b) Radial velocity fluctuation

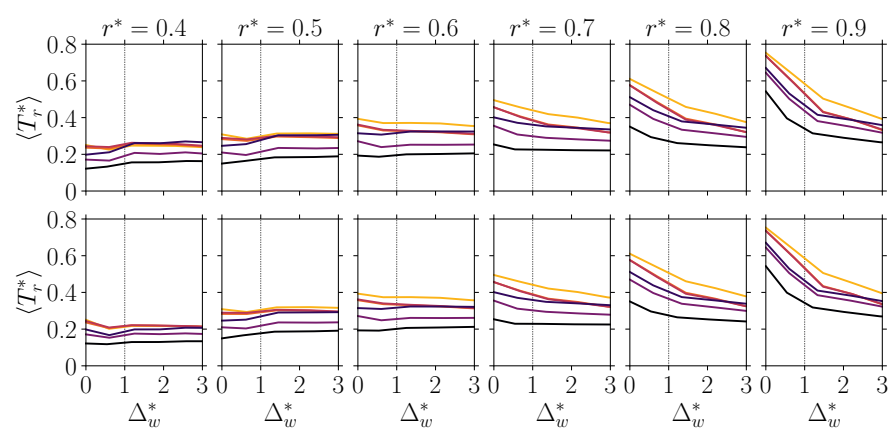

(c) Mean rotary stagnation temperature

FIGURE 13. DISC BOUNDARY LAYER PROFILES. IN EACH SUBFIGURE, TOP: UPSTREAM DISC; BOTTOM: DOWNSTREAM DISC. CURVES CORRESPOND TO CONDITIONS WITH THE SAME COLOUR IN TAB. 1.

mesh resolution there is reasonable agreement of the WMLES and analytical solutions. In the analytical solution, the far field velocity was taken to be that given by the WMLES at $\Delta_{w}^{*}=2$. The Ekman layers on both discs are in-phase. Further examination of the instantaneous velocities found that the core tangential velocity rarely exceeded disc speed.

As mentioned above the rotary stagnation temperature profile shows a sharp gradient across the disc Ekman layer. This motivates analysis of the disc heat transfer based on the Ekman layer length scale. A disc Nusselt number $N u_{d}$ is defined based on disc to bore inlet flow temperature difference and local radius.

$$
N u_{d}=\frac{\dot{Q}_{d} r}{\kappa_{d}\left(T_{r, d}-T_{a}\right)}
$$



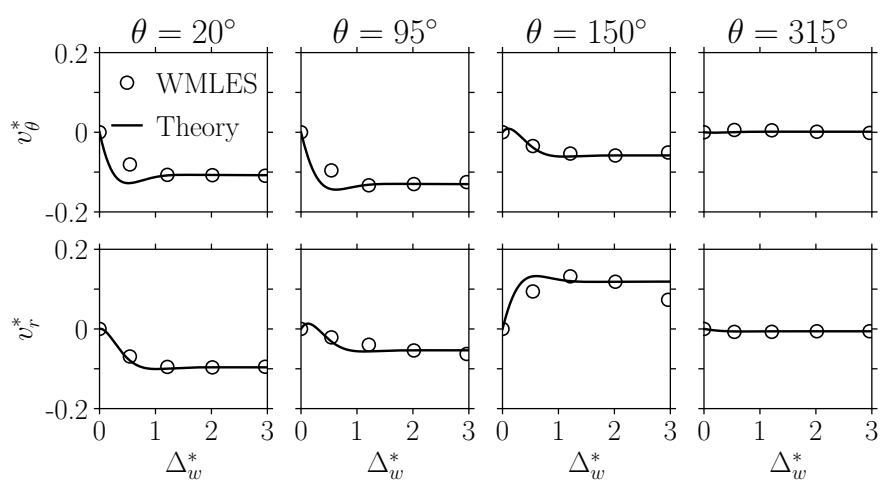

(a) Upstream disc
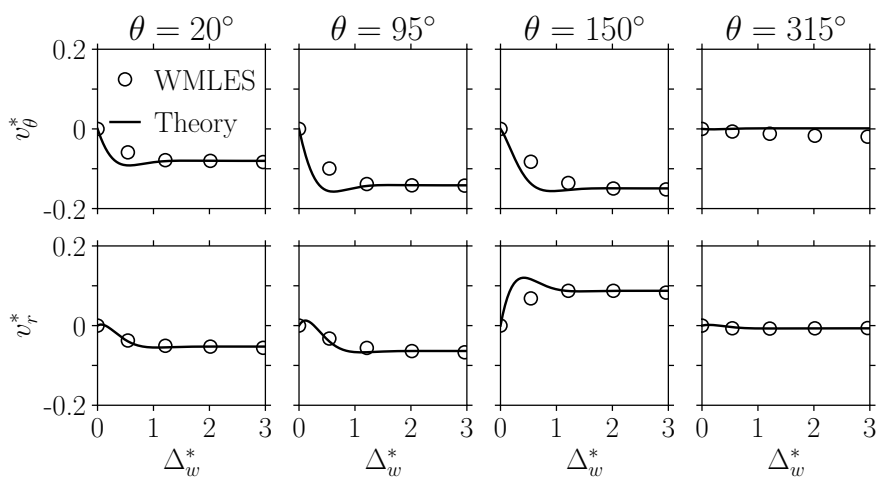

(b) Downstream disc

FIGURE 14. INSTANTANEOUS EKMAN LAYER VELOCITY PROFILES AT $r^{*}=0.85$ FOR 6000 RPM.

Values of $N u_{d}$ corresponding to conduction across the Ekman layer are obtained (for comparison to the WMLES) by setting $\dot{Q}_{d}=\kappa_{d}\left(T_{r, d}-\left\langle T_{r, \text { core }}\right\rangle\right) / \delta$ in Eq. (7). Here $T_{r, \text { core }}$ is taken as the value at $r^{*}=0.9$.

The disc Nusselt numbers $N u_{d}$ on the up and downstream discs are plotted in Fig. 15, along with auxiliary dotted curves indicating 0, 0.5 and 1 times the Ekman layer conduction. For the lowest speed and $\beta \Delta T$ condition considered the heat transfer is smaller than $0.5 \dot{Q}_{\text {cond }}$ on the outer part of the disc $\left(r^{*} \geq 0.6\right)$. Keeping the same rotational speed and increasing $\beta \Delta T$ the disc heat transfer approaches half of the Ekman layer conduction. With moderate speed (2000rpm) the disc heat transfer approximates $0.5 \dot{Q}_{\text {cond }}$ in the middle part of the disc and is close to $\dot{Q}_{\text {cond }}$ near the shroud. For the highest speed condition studied Ekman layer condition is observed over the main part of the disc. The negative $N u_{d}$ on the downstream cob corresponds to heat transfer from the fluid to the cob and implies the choice of the reference temperature is inappropriate. Further work is needed to characterise the cob heat transfer, which is likely to be more significant during engine transients than at stabilised thermal conditions.

The disc Nusselt numbers in the outer part of the cavity for the 6000rpm case are close to those for Ekman layer conduction. This corresponds to the bulk of the disc-to-core mean temperature difference occurring across the Ekman layer as shown in Fig. 13 (c). For lower speed cases there is a more significant axial temperature gradient outside the Ekman layer and the Nusselt numbers are lower. It appears that at higher Grashof numbers
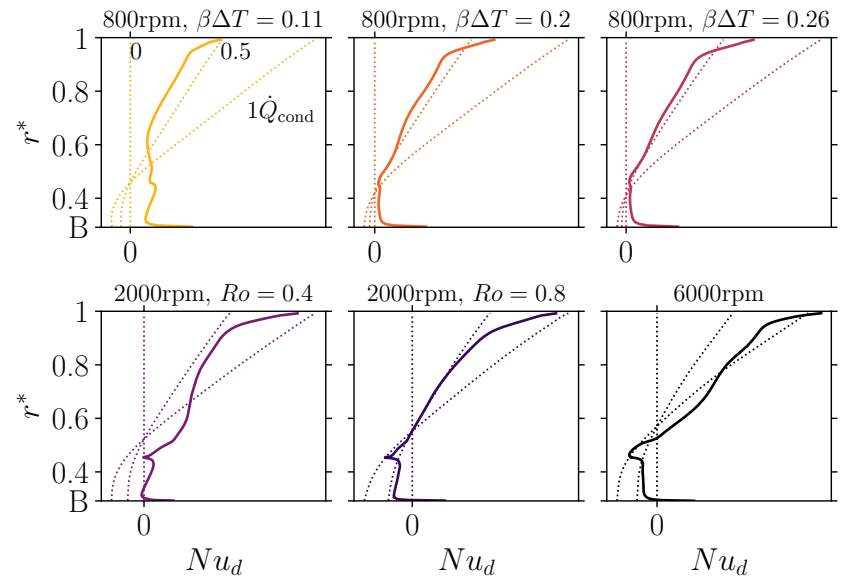

(a) Upstream
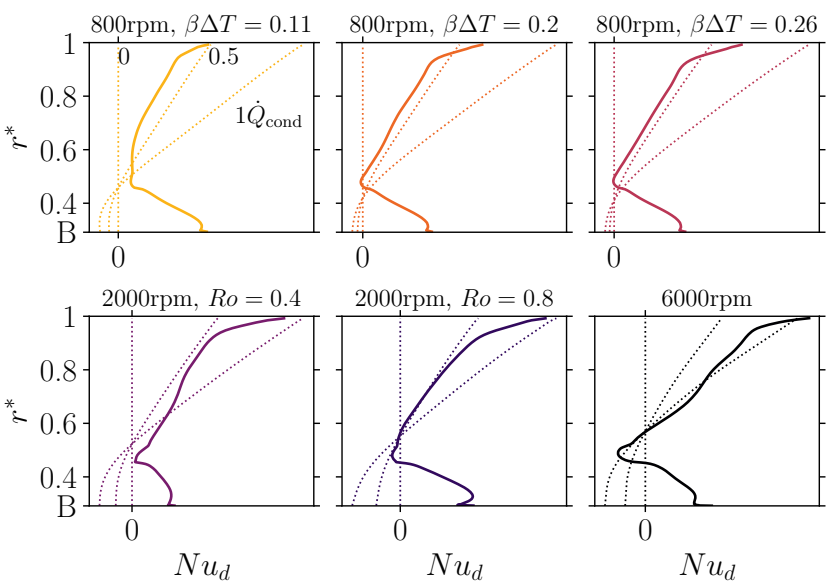

(b) Downstream

FIGURE 15. DISC NUSSELT NUMBER. DOTTED CURVES INDICATES 0, 0.5 AND 1 TIMES THE EKMAN LAYER CONDUCTION AS LABELED IN THE FIRST SUBPLOT.

the turbulent mixing in the core is sufficient to confine the axial gradient to the Ekman layer, and this is supported by comparison with experiment in Fig. 5. This interpretation is consistent with Saini and Sandberg's wall-resolved LES results [11] for a relatively low Grashof number condition. In [11] strong temperature gradients are shown across the Ekman layers at low radii. At higher radii where turbulence is much weaker there is little apparent effect of the Ekman layers on the axial temperature gradients, as has been observed in laminar theory and computations [32].

Further evidence of Ekman layer conduction governing disc heat transfer is obtained from the disc temperature and heat flux measurements given by Farthing et al. [33]. These experiments considered configurations where either both or just one of the discs was heated. Estimating core temperatures as equal to the (almost uniform) temperature measured on the unheated disc allows comparison of heat flux gauge measurements with values for Ekman layer heat conduction. An example is shown in Fig. 16. This considers the "increasing" disc temperature case and heat flux measurements from Fig. 6 (i)(a) of Farthing et al.'s paper. As for the higher Grashof number case in the present study, the disc heat transfer is close to that given by Ekman layer conduction. 


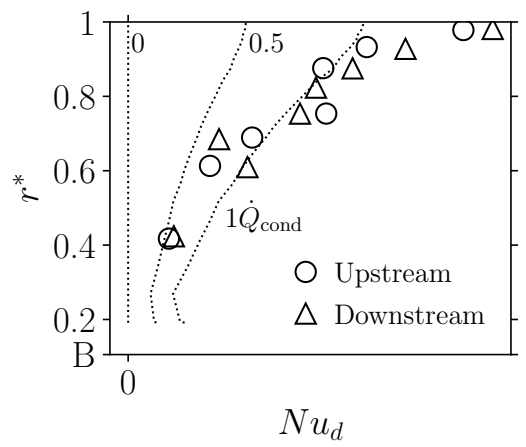

FIGURE 16. DISC NUSSELT NUMBER. REPRODUCED FROM FARTHING ET AL.'S MEASUREMENTS [33]. $\beta \Delta T=0.27, R o=$ 2.4, $R e_{\phi}=3 \times 10^{6}$.

\subsection{Bore flow}

This section discusses the characteristics of the bore flow and the disc bore heat transfer. The mean axial velocity and fluctuation profiles are plotted in Fig. 17 inboard of the upstream and downstream cobs at the positions shown in Fig. 6. There is a clear Rossby number effect separating mean axial velocity profiles at different $R o$ conditions. Close to the disc bore the axial velocity fluctuations' magnitude can be stronger than the mean, indicating that reversed flow can occur. As the Rossby number is increased the relative strength of the fluctuation compared to the mean reduces and the likelihood of reverse flow in the bore is lowered. The CFD boundary condition restricts the reverse flow, but the flow reversal may propagate more freely in the experiment. This explains the difference between the WMLES and measured temperatures of the bore flow in Fig. 6 .
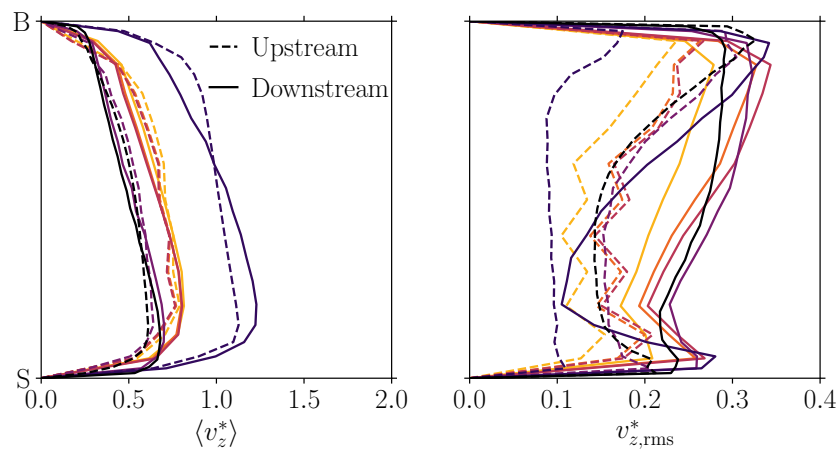

FIGURE 17. BORE FLOW VELOCITY AND FLUCTUATION PROFILES AT THE AXIAL POSITION OF THE TEMPERATURE RAKES IN FIG. 6. CURVES CORRESPOND TO CONDITIONS WITH THE SAME COLOUR IN TAB. 1.

There is little information on disc bore heat transfer in the open literature. However this is a significant factor in determining disc temperatures and the calculated bore heat fluxes for the present case are considerably higher than heat fluxes on the other surfaces of the disc cobs. Results are compared with the laminar and turbulent pipe flow forced convection correlations [34].

$$
\text { Laminar : } N u_{a}=1.83 \operatorname{Re}_{a}^{1 / 3} \operatorname{Pr}^{1 / 3}\left(l_{a} / d_{h}\right)^{-1 / 3}\left(\mu_{\text {bulk }} / \mu_{a}\right)^{0.14}
$$

$$
\text { Turbulent : } N u_{a}=0.015 \operatorname{Re}_{a}^{0.83} \operatorname{Pr}^{0.42}\left(\mu_{\text {bulk }} / \mu_{a}\right)^{0.14}
$$

The term $\left(\mu_{\text {bulk }} / \mu_{a}\right)^{0.14}$ is neglected due to small difference between the bulk and bore flow viscosities. The disc bore Nusselt number is defined by Eq. (10).

$$
N u_{a}=\frac{\dot{Q}_{a} d_{h}}{\kappa_{a}\left(T_{a}-\left\langle T_{\mathrm{bulk}}\right\rangle\right)}
$$

The WMLES calculated Nusselt numbers on both the up and downstream disc bores and the pipe flow forced convection correlations against bore flow Reynolds numbers are shown in Fig. 18. Reasonable agreement between the WMLES solution and the correlation for turbulent flow is obtained for all conditions considered in the present study.

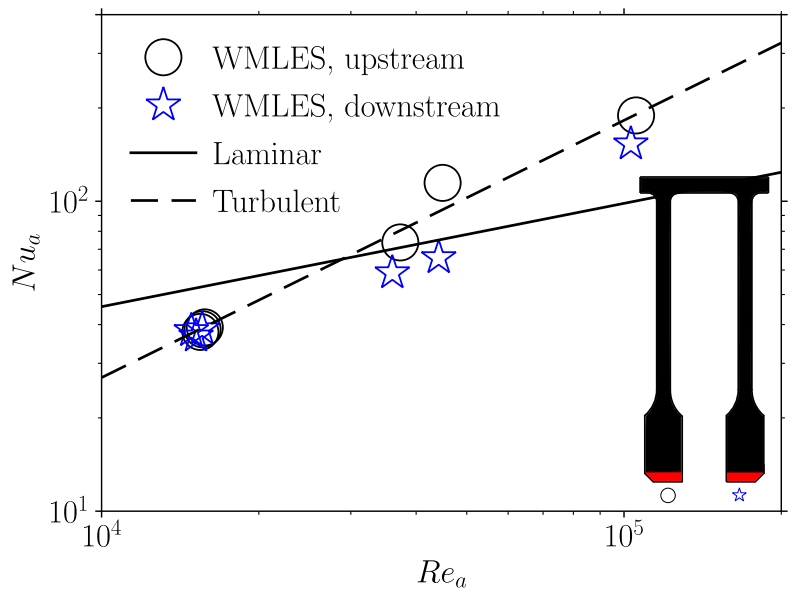

FIGURE 18. DISC BORE HEAT TRANSFER.

\subsection{Unsteady flow structure}

A number of publications have shown evidence that the flow field is dominated by unsteady flow structures with cold and hot arms in the cavity. In this paper the unsteady flow structure is illustrated through rotary stagnation temperature contours at the cavity centre for the $6000 \mathrm{rpm}$ condition in Fig. 19. Four representative time instants are selected to show unsteady flow structures at different phases. For the majority of the time the flow structure has one lobe. At the 29.5th rotor revolution (in subplot (c)) a second lobe emerges but merges quickly with the main lobe 3 revolutions later. Some small hot plumes can be observed at the shroud, in agreement with the observation of RayleighBénard convection cells reported, for example, by Saini and Sandberg [11] and Puttock-Brown et al. [7]. Referring back to Fig. 14 confirms that axial gradients in the core flow are small with adjustment to no-slip conditions on the discs through the Ekman layers.

The drifting speed of the unsteady flow structure can be calculated through cross correlation of pressure signals taken on circumferentially distributed numerical probes. The angular speed of the flow structure $\omega_{s}$ varies between $84-89 \% \Omega$ for the conditions studied in this paper, slower than the disc rotational speed. The rotating flow structure was investigated by setting a rotating speed $\Omega-\omega_{s}$ to the grid, during the post-processing. The corresponding phase locked flow contours are shown in the bottom 


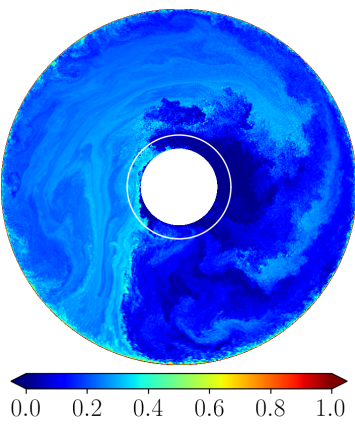

(a) $0.5 \mathrm{rev}$.

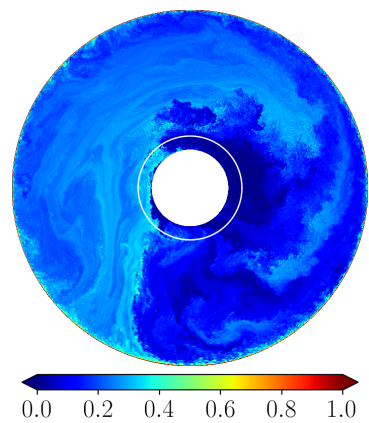

(e) $0.5 \mathrm{rev}$.

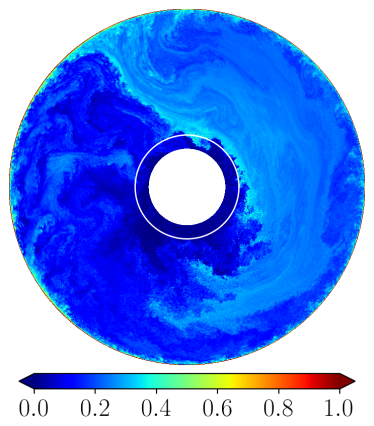

(b) $2.5 \mathrm{rev}$.

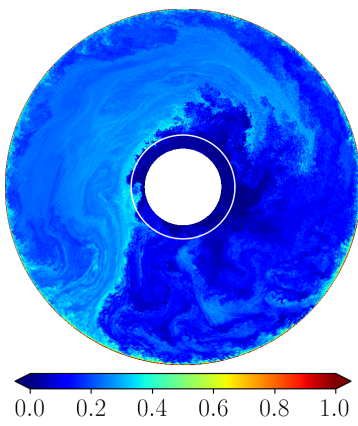

(f) 2.5 rev.

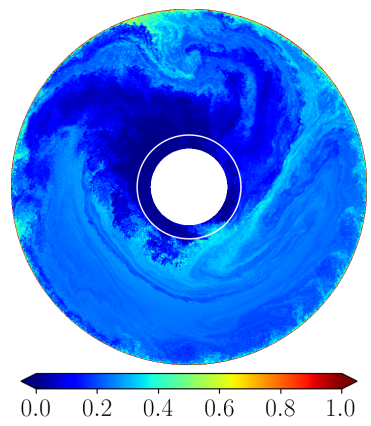

(c) $29.5 \mathrm{rev}$.

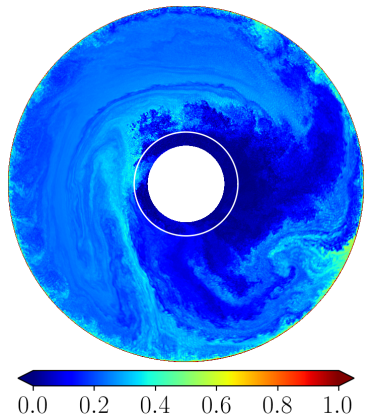

(g) $29.5 \mathrm{rev}$.

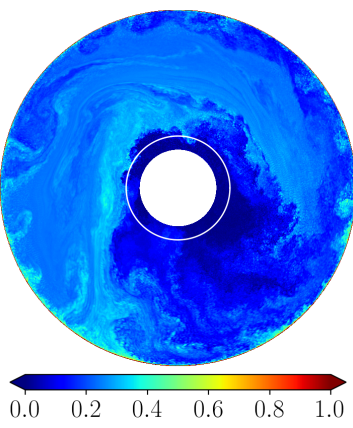

(d) $32.5 \mathrm{rev}$.

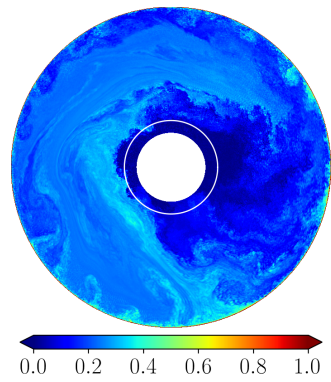

(h) $32.5 \mathrm{rev}$

FIGURE 19. INSTANTANEOUS ROTARY STAGNATION TEMPERATURE CONTOURS FOR 6000 RPM AT THE MID-AXIAL POSITION OF THE CAVITY. VIEW FROM DOWNSTREAM. (a)-(d) IN THE DISC REFERENCE FRAME. (e)-(h) IN THE REFERENCE FRAME OF THE FLOW STRUCTURE. THE ROTATION OF THE DISCS IS ANTI-CLOCKWISE.

row of Fig. 19. These results represent those in the top row as seen by an observer rotating at speed $\Omega-\omega_{s}$. Some vacillation is apparent with the cold flow lobe oscillating over a short circumferential extent.

To further analyse the unsteady flow feature flow variables were averaged in the reference frame of the flow structure. These are shown in Fig. 20. The averaged rotary stagnation temperature contour shows hot and cold lobes initiated from the shroud and throughflow, respectively, and spreading in the clockwise direction. The mean tangential velocity contours show a tendency of the hot fluid to rotate faster than the cooler fluid. Clear radial inflow and outflow regions are observed through the mean radial velocity contour. These velocity contours are the basis for the streamlines superimposed on the plots. Finally, low and high pressure areas are shown within the anti-cyclonic and cyclonic vortices.

The mass flow exchange between the bore flow and the cavity is of particular interest as this determines the core temperature and heat that can be extracted from the cavity. This is calculated here by integrating the instantaneous radially outward mass flow over the cavity mouth area and then averaging in time. The results are given in Tab. 4. The mass flow exchange is significant and can be as much as $42.6 \%$ of the bore flow. As a fraction of bore flow the exchange reduces when the Rossby number increases. The exchange mass flow rate normalised with the shroud radius, reference density and $\Omega a \sqrt{\beta \Delta T_{r}}$ is also given in the ta-

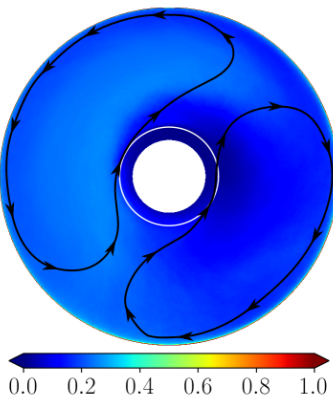

(a) $\left\langle T_{r}^{*}\right\rangle_{\mathrm{rot}}$

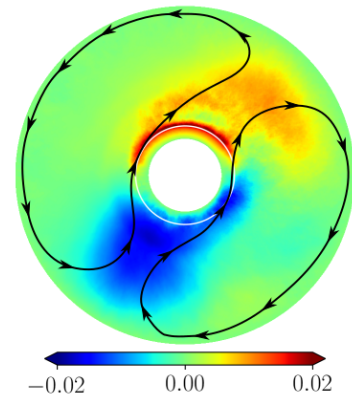

(c) $\left\langle v_{r}^{*}\right\rangle_{\text {rot }}$

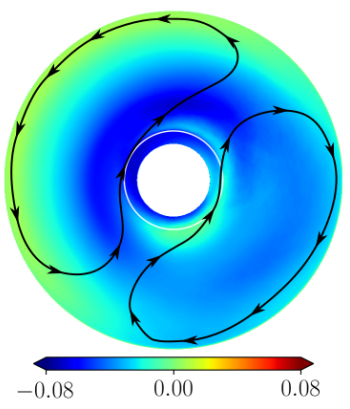

(b) $\left\langle v_{\theta}^{*}\right\rangle_{\text {rot }}$

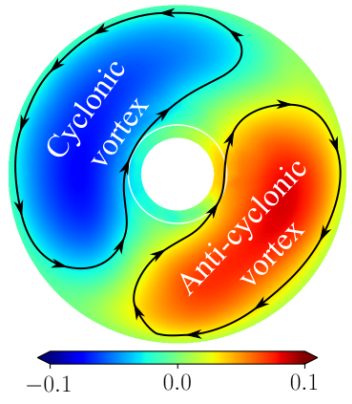

(d) $\left\langle\mathscr{C}_{p}^{*}\right\rangle_{\text {rot }}$
FIGURE 20. AVERAGED VARIABLES IN THE REFERENCE FRAME OF THE FLOW STRUCTURE FOR 6000 RPM. DISCS ROTATE IN THE ANTI-CLOCKWISE DIRECTION. 
ble. It should be noted that taking the average prior to integrating the mass flow rate will results in spuriously lower mass flow exchange. That is because the unsteady effect dominates the mean, and averaging in advance can smooth out the dominating fluctuations.

TABLE 4. MASS FLOW EXCHANGE BETWEEN THE BORE FLOW AND THE CAVITY.

\begin{tabular}{llllll}
\hline Code & $\Omega[\mathrm{rpm}]$ & $\beta \Delta T_{r}$ & $R o$ & $\dot{m}_{\mathrm{ex}} / \dot{m}$ & $\frac{\dot{m}_{\mathrm{ex}}}{\rho b^{2} \Omega a \sqrt{\beta \Delta T_{r}}}$ \\
\hline 800B11 & 790 & 0.11 & 0.5 & $26.4 \%$ & 0.050 \\
800B20 & 790 & 0.2 & 0.5 & $30.7 \%$ & 0.041 \\
800B26 & 790 & 0.26 & 0.5 & $32.2 \%$ & 0.037 \\
2kR4 & 2000 & 0.25 & 0.4 & $41.2 \%$ & 0.041 \\
2kR8 & 2000 & 0.25 & 0.8 & $17.1 \%$ & 0.033 \\
6kR4 & 6000 & 0.2 & 0.4 & $42.6 \%$ & 0.046 \\
\hline
\end{tabular}

\section{CONCLUDING REMARKS}

This paper presents a study of buoyancy induced flows in a single compressor rotating cavity. Six operating conditions are considered, spanning ranges of rotational Reynolds number $\left(3.2 \times 10^{5}-2.2 \times 10^{6}\right)$, buoyancy parameter $(0.11-0.26)$ and Rossby number $(0.4-0.8)$.

The numerical modelling techniques used are based on previous evaluations of WMLES for disc cavity flows which include predictions for rotational free convection in a sealed cavity. Accuracy is considered through balance checks and comparison with measured data. The WMLES results show remarkable agreement with the measurements. Overall balances of mass, momentum and energy in the simulations were generally considered satisfactory, with the level of energy imbalance being comparable to the differences between measurements and simulations.

The flow and heat transfer mechanisms occurring in a rotating compressor cavity with axial throughflow under buoyancydriven convection are summarised in Fig. 21. This shows the dominant large scale flow structure that rotates at a different speed than the rotor, the time averaged flow, and the dominant heat transfer mechanisms on the shroud, disc webs and disc bores.
In the core of the cavity a negative slip velocity was found for all conditions considered in this study. This produced a weak mean radial outflow in the disc boundary layers, as indicated in the fully averaged description in Fig. 21. The velocity fluctuations scale with $\Omega r \sqrt{\beta \Delta T}$ at the outer part of the cavity where buoyancy dominates. The flow near the discs is characterised by unsteady, laminar Ekman layers, whilst that in the core of the cavity is turbulent.

The time-averaged core flow in the cavity can be considered well-mixed, resulting in uniform mean rothalpy. At high speeds the Eckert number becomes significant and rotary stagnation temperature should be considered, instead of static temperature, in heat transfer calculations.

The correlation $N u_{b}=0.057 R a^{0.366}$ has been obtained for shroud heat transfer, in good agreement with the $N u \sim R a$ scaling for natural convection between horizontal plates under gravity at high $R a$ numbers. The heat transfer from the disc webs is largely controlled by conduction across the Ekman layers. For the stabilised thermal conditions considered here the heat transfer on the disc cob faces is relatively weak. The downstream cob face is subject to flow impingement effects. The disc bore heat transfer was found to be reasonably close to a correlation for turbulent pipe flow forced convection.

An unsteady flow structure rotating between $84-89 \% \Omega$ was identified for all conditions studied. One pair of vortices dominates the mass and heat exchange between the cavity and bore flows and a second plume emerged intermittently. RayleighBénard convection cells appear near the shroud, with a length scale smaller than that of the main vortices.

Radial transport and exchange of mass and energy between the bore flow and the cavity are dominated by unsteady effects. The fluid moves radially in and outwards at different circumferential positions and throughout the axial extent of the cavity. Mass exchange between the bore and cavity flows was found to be up to $42.6 \%$ of the bore flow rate.

This study provides a systematic overview of the flow and heat transfer mechanisms in a buoyancy-driven compressor drum cavity and builds up confidence in using high-fidelity numerical methods. The results obtained could be used to establish thermal boundary conditions to thermo-mechanical finite element models and the heat transfer scaling could help to choose the most appro-

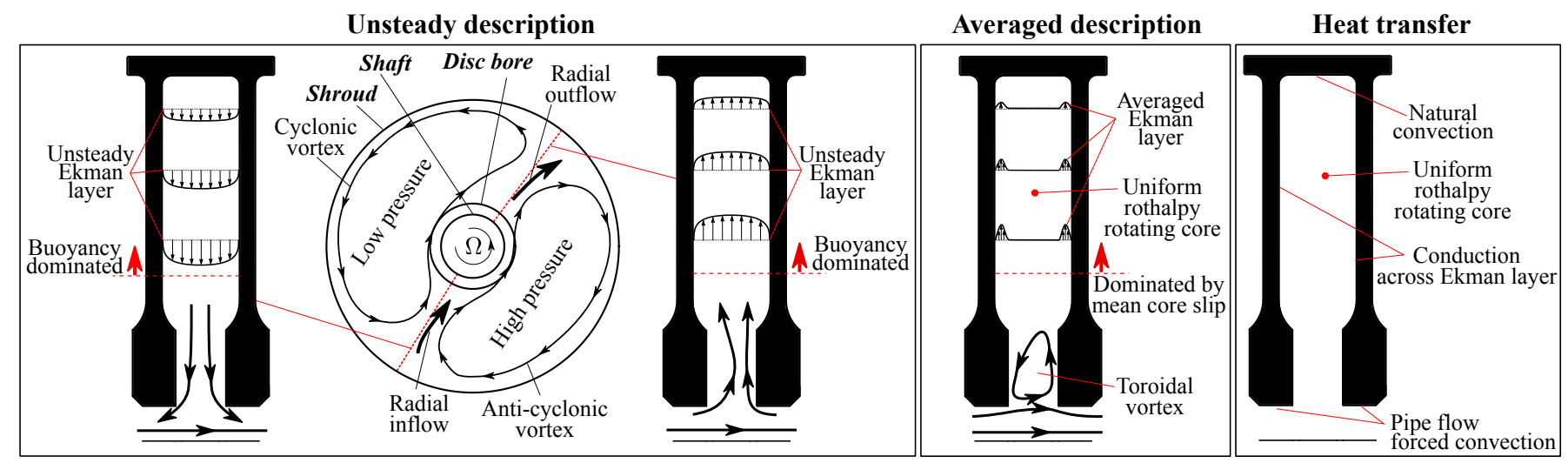

FIGURE 21. SUMMARY OF FLOW AND HEAT TRANSFER MECHANISMS. 
priate finite element heat transfer model coefficients, to improve the design tool and lower the risk level of redesign [35].

Difficulties in stabilising simulations at low Rossby number ( $R o=0.2, \Omega=8000 \mathrm{rpm}$ ) have been identified. These are associated with the reverse flow at inlet and outlet boundaries, induced by the pressure asymmetry due to the unsteady flow structure in the cavity. Future research should consider interaction between cavities, as may be expected in multiple-cavity and real engine configurations.

\section{ACKNOWLEDGMENT}

The authors are grateful for the financial support provided by the Engineering and Physical Sciences Research Council under grant EP/P004091 as part of a joint project with the University of Bath. We thank colleagues at the Universities of Surrey and Bath and Rolls-Royce plc for their technical support. The computing resources from ARCHER and local clusters in the University of Surrey are much appreciated.

\section{REFERENCES}

[1] Atkins, N. R., 2013. "Investigation of a radial-inflow bleed as a potential for compressor clearance control”. ASME Paper No. GT2013-95768.

[2] Bohn, D., Deuker, E., Emunds, R., and Gorzelitz, V., 1995. "Experimental and theoretical investigations of heat transfer in closed gas-filled rotating annuli". ASME J. Turbomach., 117(1), pp. 175-183.

[3] Gao, F., and Chew, J. W., 2021. "Ekman layer scrubbing and shroud heat transfer in centrifugal buoyancy-driven convection". ASME J. Eng. Gas Turbines Power, 143(7), p. 071010. Also ASME Paper No. GT2020-16220.

[4] Owen, J. M., and Long, C. A., 2015. "Review of buoyancy-induced flow in rotating cavities". ASME J. Turbomach., 137(11), p. 111001.

[5] Pitz, D. B., Marxen, O., and Chew, J. W., 2017. "Onset of convection induced by centrifugal buoyancy in a rotating cavity". J. Fluid Mech., 826, pp. 484-502.

[6] Pitz, D. B., Chew, J. W., and Marxen, O., 2018. "Large-eddy simulation of buoyancy-induced flow in a sealed rotating cavity". ASME J. Eng. Gas Turbines Power, 141(2), p. 021020.

[7] Puttock-Brown, M. R., Rose, M. G., and Long, C. A., 2017. "Experimental and computational investigation of Rayleigh-Bénard flow in the rotating cavities of a core compressor". ASME Paper No. GT2017-64884.

[8] Tateishi, A., Watanabe, T., and Himeno, T., 2018. "Unsteady flow simulation of buoyancy-driven flows in high-pressure compressor disk cavities". ASME Paper No. GT2018-76327.

[9] Gao, F., Pitz, D. B., and Chew, J. W., 2020. "Numerical investigation of buoyancy-induced flow in a sealed rapidly rotating disc cavity". Int. J. Heat Mass Transfer, 147, p. 118860.

[10] Jackson, R. W., Luberti, D., Tang, H., Pountney, O. J., Scobie, J. A., Sangan, C. M., Owen, J. M., and Lock, G. D., 2020. "Measurement and analysis of buoyancy-induced heat transfer in aero-engine compressor rotors". ASME Paper No. GT2020-14427.

[11] Saini, D., and Sandberg, R. D., 2020. "Large eddy simulations of high Rossby number flow in the high pressure compressor inter-disk cavity". ASME Paper No. GT2020-14463.

[12] Jiang, H., Zhu, X., Wang, D., Huisman, S. G., and Sun, C., 2020. "Supergravitational turbulent thermal convection". Sci. Adv., 6(40), p. eabb8676.

[13] Saini, D., and Sandberg, R. D., 2020. "Simulations of compressibility effects in centrifugal buoyancy-induced flow in a closed rotating cavity". Int. J. Heat Fluid Flow, 85, p. 108656.

[14] Tang, H., Puttock-Brown, M. R., and Owen, M. J., 2018. "Buoyancyinduced flow and heat transfer in compressor rotors". ASME J. Eng. Gas Turbines Power, 140(7), p. 071902.

[15] Owen, J. M., Tang, H., and Lock, G. D., 2018. "Buoyancy-induced heat transfer inside compressor rotors: overview of theoretical models". Aerospace, 5(1), p. 32.

[16] Pitz, D. B., Chew, J. W., Marxen, O., and Hills, N. J., 2017. "Direct numerical simulation of rotating cavity flows using a spectral element-Fourier method". ASME J. Eng. Gas Turbines Power, 139(7), p. 072602.

[17] Pitz, D. B., Chew, J. W., and Marxen, O., 2019. "Effect of an axial throughflow on buoyancy-induced flow in a rotating cavity". Int. J. Heat Fluid Flow, 80, p. 108468.

[18] Luberti, D., Patinios, M., Jackson, R. W., Tang, H., Pountney, O. J., Scobie, J. A., Sangan, C. M., Owen, J. M., and Lock, G. D., 2020. "Design and testing of a rig to investigate buoyancy-induced heat transfer in aero-engine compressor rotors". ASME Paper No. GT2020-14422.

[19] Long, C. A., Miché, N. D. D., and Childs, P. R. N., 2007. "Flow measurements inside a heated multiple rotating cavity with axial throughflow". Int. J. Heat Fluid Flow, 28(6), pp. 1391-1404.

[20] Sun, Z., Lindblad, K., Chew, J. W., and Young, C., 2006. "LES and RANS investigations into buoyancy-affected convection in a rotating cavity with a central axial throughflow". ASME J. Eng. Gas Turbines Power, 129(2), pp. 318-325.

[21] Long, C. A., and Childs, P. R. N., 2007. "Shroud heat transfer measurements inside a heated multiple rotating cavity with axial throughflow". Int. J. Heat Fluid Flow, 28(6), pp. 1405-1417.

[22] Farthing, P. R., Long, C. A., Owen, J. M., and Pincombe, J. R., 1992. "Rotating cavity with axial throughflow of cooling air: flow structure". ASME J. Turbomach., 114(1), pp. 237-246.

[23] Sun, Z., Amirante, D., Chew, J. W., and Hills, N. J., 2016. "Coupled aerothermal modeling of a rotating cavity with radial inflow". ASME J. Eng. Gas Turbines Power, 138(3), p. 032505.

[24] Amirante, D., and Hills, N. J., 2015. "Large-eddy simulations of wall bounded turbulent flows using unstructured linear reconstruction techniques". ASME J. Turbomach., 137(5), p. 051006.

[25] Spalding, D. B., 1961. "A single formula for the "law of the wall"”. ASME J. Appl. Mech., 28(3), pp. 455-458.

[26] Palermo, D. M., Gao, F., Amirante, D., Chew, J. W., Bru-Revert, A., and Beard, P. F., 2021. "Wall-modelled large eddy simulations of axial turbine rim sealing". ASME J. Eng. Gas Turbines Power, 143(6), p. 061025. Also ASME Paper No. GT2020-14973.

[27] Gao, F., and Chew, J. W., 2021. "Evaluation and application of advanced CFD models for rotating disc flows". Proc. Inst. Mech. Eng. Part C-J. Mech. Eng. Sci. Accepted.

[28] Jackson, R. W., and Lock, G. D., 2020. Private Communication.

[29] Jackson, R. W., Tang, H., Scobie, J. A., Pountney, O. J., Sangan, C. M., Owen, J. M., and Lock, G. D., 2021. "Unsteady pressure measurements in a heated rotating cavity". ASME Paper No. GT2021-59090.

[30] Miché, N. D. D., 2009. "Flow and heat transfer measurements inside a heated multiple rotating cavity with axial throughflow". PhD thesis, University of Sussex.

[31] Wu, C.-H., 1953. "Discussion of "A practical solution of a threedimensional flow problem of axial flow turbomachinery" by Smith, LH, Traugott, SC, and Wislicenus, GF". ASME Transactions, 75(1), pp. 802803.

[32] Chew, J. W., 1985. "Computation of convective laminar flow in rotating cavities". J. Fluid Mech., 153, pp. 339-360.

[33] Farthing, P. R., Long, C. A., Owen, J. M., and Pincombe, J. R., 1992. "Rotating cavity with axial throughflow of cooling air: heat transfer". Journal of Turbomachinery, 114(1), pp. 229-236.

[34] Whitaker, S., 1972. "Forced convection heat transfer correlations for flow in pipes, past flat plates, single cylinders, single spheres, and for flow in packed beds and tube bundles". AIChE J., 18(2), pp. 361-371.

[35] Dixon, J. A., Verdicchio, J. A., Benito, D., Karl, A., and Tham, K. M., 2004. "Recent developments in gas turbine component temperature prediction methods, using computational fluid dynamics and optimization tools, in conjunction with more conventional finite element analysis techniques". Proc. Inst. Mech. Eng. Part A-J. Power Energy, 218(4), pp. 241-255. 